REG 174 INFORMATION FOR UK HEALTHCARE PROFESSIONALS 
This medicinal product has been given authorisation for temporary supply by the UK Department of Health and Social Care and the Medicines \& Healthcare products Regulatory Agency. It does not have a marketing authorisation, but this temporary authorisation grants permission for the medicine to be used for active immunisation to prevent COVID-19 disease caused by SARS-CoV-2 virus in individuals aged 12 years of age and over.

As with any new medicine in the UK, this product will be closely monitored to allow quick identification of new safety information. Healthcare professionals are asked to report any suspected adverse reactions. See section 4.8 for how to report adverse reactions.

\section{NAME OF THE MEDICINAL PRODUCT}

COVID-19 mRNA Vaccine BNT162b2 concentrate for solution for injection

\section{QUALITATIVE AND QUANTITATIVE COMPOSITION}

This is a multidose vial and must be diluted before use. 1 vial $(0.45 \mathrm{~mL})$ contains 6 doses of 30 micrograms of tozinameran, a BNT162b2 RNA (embedded in lipid nanoparticles), see section 4.2.

Tozinameran is highly purified single-stranded, 5'-capped messenger RNA (mRNA) produced by cellfree in vitro transcription from the corresponding DNA templates, encoding the viral spike (S) protein of SARS-CoV-2.

Excipients with known effect:

For the full list of excipients, see section 6.1.

\section{PHARMACEUTICAL FORM}

Concentrate for solution for injection.

The vaccine is a white to off-white frozen solution.

\section{CLINICAL PARTICULARS}

\subsection{Therapeutic indications}

Active immunisation to prevent COVID-19 caused by SARS-CoV-2 virus, in individuals 12 years of age and older.

The use of COVID-19 mRNA Vaccine BNT162b2 should be in accordance with official guidance.

\subsection{Posology and method of administration}

\section{Posology}

Individuals 12 years of age and older

COVID-19 mRNA Vaccine BNT162b2 is administered intramuscularly after dilution as a series of two doses $(0.3 \mathrm{~mL}$ each) at least 21 days apart (see section 5.1).

There are no data available on the interchangeability of COVID-19 mRNA Vaccine BNT162b2 with other COVID-19 vaccines to complete the vaccination series. Individuals who have received one dose of COVID-19 mRNA Vaccine BNT162b2 should receive a second dose of COVID-19 mRNA Vaccine BNT162b2 to complete the vaccination series.

Individuals may not be maximally protected until at least 7 days after their second dose of the vaccine. 
One dose of COVID-19 mRNA Vaccine BNT162b2 may be administered as a third dose at least 8 weeks after the second dose of an mRNA or adenovirus-vectored COVID-19 vaccine when the potential benefits outweigh any potential risks.

For further information on efficacy, see section 5.1.

\section{Paediatric population}

The safety and efficacy of COVID-19 mRNA Vaccine BNT162b2 in children under 12 years of age have not yet been established.

\section{Method of administration}

Administer the COVID-19 mRNA Vaccine BNT162b2 intramuscularly in the deltoid muscle after dilution.

After dilution, vials of COVID-19 mRNA Vaccine BNT162b2 contain six doses of $0.3 \mathrm{~mL}$ of vaccine. In order to extract six doses from a single vial, low dead-volume syringes and/or needles should be used. If standard syringes and needles are used, there may not be sufficient volume to extract a sixth dose from a single vial. Irrespective of the type of syringe and needle:

- $\quad$ Each dose must contain $0.3 \mathrm{~mL}$ of vaccine.

- If the amount of vaccine remaining in the vial cannot provide a full dose of $0.3 \mathrm{~mL}$, discard the vial and any excess volume.

- Do not pool excess vaccine from multiple vials.

Preparation: The multidose vial is stored frozen and must be thawed prior to dilution.

Frozen vials should be transferred to $2^{\circ} \mathrm{C}$ to $8^{\circ} \mathrm{C}$ to thaw.

Alternatively, frozen vials may also be thawed and kept at temperatures up to $25^{\circ} \mathrm{C}$ for a maximum of two hours in preparation for dilution for use.

When removed from the freezer, the undiluted vaccine has a maximum shelf life of up to 1 month (31 days) at $2^{\circ} \mathrm{C}$ to $8^{\circ} \mathrm{C}$ and an additional 2 hours at temperatures up to $25^{\circ} \mathrm{C}$ in preparation for dilution.

When the thawed vial is at room temperature gently invert 10 times prior to dilution. Do not shake.

Prior to dilution, the thawed dispersion may contain white to off-white opaque amorphous particles.

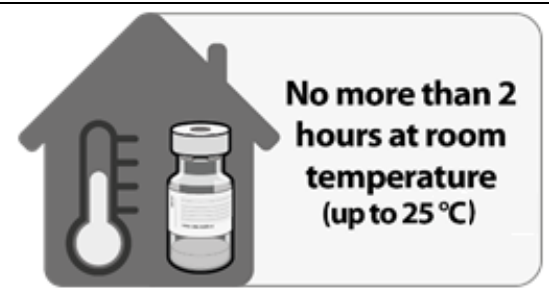




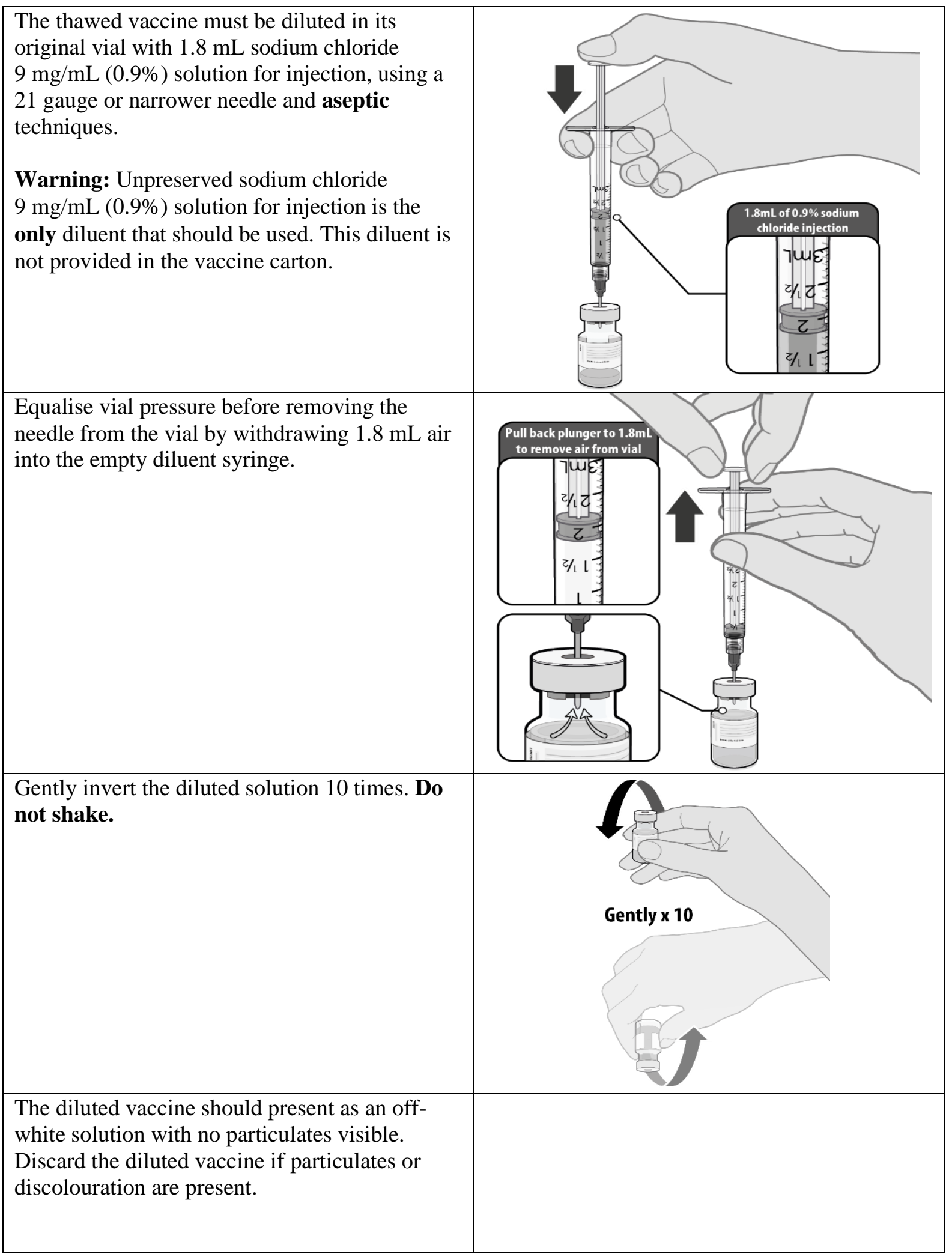




The diluted vials should be marked with the
dilution date and time and stored between $2^{\circ} \mathrm{C}$
to $25^{\circ} \mathrm{C}$.
Use as soon as practically possible, and within 6
hours after dilution.
After dilution, the vial contains 6 doses of
0.3 mL.
Withdraw the required $0.3 \mathrm{~mL}$ dose of diluted
vaccine using a sterile needle and syringe and
administer.
Low dead-volume syringes and/or needles
should be used in order to extract 6 doses from a
single vial.
If standard syringes and needles are used, there
may not be sufficient volume to extract a sixth
dose from a single vial.
Each dose must contain $0.3 \mathrm{~mL}$ of vaccine.
If the amount of vaccine remaining in the vial
cannot provide a full dose of $0.3 \mathrm{~mL}$, discard the
vial and any excess volume.
Any unused vaccine should be discarded
6 hours after dilution.
The vaccine should not be shipped (transported)
by motor vehicle after dilution away from the
site of dilution. Any shipping (transportation) by
motor vehicle after dilution of the vial is at the
risk of the Health Care Professional.

For instructions on disposal see section 6.6.

\subsection{Contraindications}

Hypersensitivity to the active substance or to any of the excipients listed in section 6.1 .

\subsection{Special warnings and precautions for use}

$\underline{\text { Hypersensitivity and anaphylaxis }}$

Events of anaphylaxis have been reported. Appropriate medical treatment and supervision should always be readily available in case of an anaphylactic reaction following the administration of the vaccine.

Close observation for at least 15 minutes is recommended following vaccination. A second dose of the vaccine should not be given to those who have experienced anaphylaxis to the first dose of the COVID-19 mRNA Vaccine BNT162b2. 


\section{Myocarditis and pericarditis}

There is an increased risk of myocarditis and pericarditis occurring after vaccination with COVID-19 mRNA Vaccine BNT162b2. These conditions can develop within just a few days after vaccination, and have primarily occurred within 14 days. They have been observed more often after the second vaccination, and more often in younger males (see section 4.8). Available data suggest that the course of myocarditis and pericarditis following vaccination is not different from myocarditis or pericarditis in general.

Healthcare professionals should be alert to the signs and symptoms of myocarditis and pericarditis. Vaccinated individuals (including parents or caregivers) should also seek immediate medical attention should they experience new onset of chest pain, shortness of breath, palpitations or arrhythmias following vaccination.

\section{Traceability}

In order to improve the traceability of biological medicinal products, the name and the batch number of the administered product should be clearly recorded.

\section{General recommendations}

The administration of COVID-19 mRNA Vaccine BNT162b2 should be postponed in individuals suffering from acute severe febrile illness. The presence of a minor infection and/or low-grade fever should not delay vaccination.

Anxiety-related reactions, including vasovagal reactions (syncope), hyperventilation or stress-related reactions (e.g. dizziness, palpitations, increases in heart rate, alterations in blood pressure, tingling sensations and sweating) may occur in association with the vaccination process itself. Stress-related reactions are temporary and resolve on their own. Individuals should be advised to bring symptoms to the attention of the vaccination provider for evaluation. It is important that precautions are in place to avoid injury from fainting.

Individuals receiving anticoagulant therapy or those with a bleeding disorder that would contraindicate intramuscular injection, should not be given the vaccine unless the potential benefit clearly outweighs the risk of administration.

Immunocompromised persons, including individuals receiving immunosuppressant therapy, may have a diminished immune response to the vaccine. No data are available about concomitant use of immunosuppressants.

From an independent report (Kamar N, Abravanel F, Marion O, et al. Three doses of an mRNA Covid19 vaccine in solid-organ transplant recipients. $N$ Engl J Med. 2021), safety and effectiveness of a third dose of COVID-19 mRNA Vaccine BNT162b2 have been evaluated in persons that received solid organ transplants. The administration of a third dose of vaccine appears to be only moderately effective in increasing potentially protective antibody titres. Patients should still be counselled to maintain physical precautions to help prevent COVID-19.

As with any vaccine, vaccination with COVID-19 mRNA Vaccine BNT162b2 may not protect all vaccine recipients.

No data are available on the use of COVID-19 mRNA Vaccine BNT162b2 in persons that have previously received a full or partial vaccine series with another COVID-19 vaccine.

\section{Excipient information}

This vaccine contains potassium, less than $1 \mathrm{mmol}(39 \mathrm{mg})$ per dose, i.e. essentially 'potassium-free'. This vaccine contains less than $1 \mathrm{mmol}$ sodium $(23 \mathrm{mg})$ per dose, i.e. essentially 'sodium-free'.

\subsection{Interaction with other medicinal products and other forms of interaction}

No interaction studies have been performed. 
Concomitant administration of COVID-19 mRNA Vaccine BNT162b2 with other vaccines has not been studied in trials conducted by Pfizer/BioNTech (see section 5.1).

Data assessed by the MHRA that support concomitant administration of COVID-19 mRNA Vaccine BNT162b2 with influenza vaccines (but at separate injection sites) are based on the ComFluCOV study [EudraCT Number: 2021-001124-18\}, which investigated concomitant administration of COVID-19 mRNA Vaccine BNT162b2 with several influenza vaccines. The data show that the antibody responses are unaffected and that the reactogenicity profile is acceptable. The MHRA has needed to rely on these data in advance of them being publicly available, including to Pfizer/BioNTech, but is satisfied as to the arrangements for its expected publication, and this section will be updated once the data are published.

Do not mix COVID-19 mRNA Vaccine BNT162b2 with other vaccines/products in the same syringe.

\subsection{Fertility, pregnancy and lactation}

\section{Pregnancy}

There is limited experience with use of the COVID-19 mRNA Vaccine BNT162b2 in pregnant women. Animal studies do not indicate direct or indirect harmful effects with respect to pregnancy, embryo/foetal development, parturition or post-natal development (see section 5.3). Administration of the COVID-19 mRNA Vaccine BNT162b2 in pregnancy should only be considered when the potential benefits outweigh any potential risks for the mother and foetus.

\section{Breast-feeding}

It is unknown whether the COVID-19 mRNA Vaccine BNT162b2 is excreted in human milk.

Fertility

Animal studies do not indicate direct or indirect harmful effects with respect to reproductive toxicity (see section 5.3)

\subsection{Effects on ability to drive and use machines}

COVID-19 mRNA Vaccine BNT162b2 has no or negligible influence on the ability to drive and use machines. However, some of the adverse reactions mentioned under section 4.8 may temporarily affect the ability to drive or use machines.

\subsection{Undesirable effects}

Summary of safety profile

The safety of COVID-19 mRNA Vaccine BNT162b2 was evaluated in participants 12 years of age and older in two clinical studies that included 23,205participants (comprised of 22,074participants 16 years of age and older and 1,131 adolescents 12 to 15 years of age) that have received at least one dose of COVID-19 mRNA Vaccine BNT162b2. Study BNT162-01 (Study 1) enrolled 60 participants, 18 through 55 years of age. Study C4591001 (Study 2) enrolled approximately 44,000 participants, 12 years of age or older.

\section{Participants 16 years of age and older}

In Study 2, a total of 22,026participants 16 years of age or older received at least one dose of COVID19 mRNA Vaccine BNT162b and 22,021participants 16 years of age or older received placebo. A total of 20,519 participants 16 years of age or older received 2 doses of COVID-19 mRNA Vaccine BNT162b.

At the time of the analysis of Study 2 with a data cut-off of 13 March 2021 for the placebo-controlled blinded follow-up period up to the participants' unblinding dates, a total of 25,651 (58.2\%) participants (13,031 COVID-19 mRNA Vaccine BNT162b2 and 12,620 placebo) 16 years of age and older were followed up for $\geq 4$ months after the second dose. This included a total of $15,111(7,704$ 
COVID-19 mRNA Vaccine BNT162b2 and 7,407 placebo) participants 16 to 55 years of age and a total of 10,540 (5,327 COVID-19 mRNA Vaccine BNT162b2 and 5,213 placebo) participants 56 years of age and older.

Demographic characteristics were generally similar with regard to age, gender, race and ethnicity among participants who received COVID-19 mRNA Vaccine and those who received placebo. Overall, among the participants who received COVID-19 mRNA Vaccine BNT162b2, 51.5\% were male and $48.5 \%$ were female, $82.1 \%$ were White, 9.6\% were Black or African American, $26.1 \%$ were Hispanic/Latino, $4.3 \%$ were Asian and $0.7 \%$ were Native American/Alaskan native.

The most frequent adverse reactions in participants 16 years of age and older were pain at the injection site (> 80\%), fatigue (> 60\%), headache (> 50\%), myalgia (> 40\%), chills (> 30\%), arthralgia (> 20\%), pyrexia and injection site swelling $(>10 \%)$.

The safety profile in 545 subjects receiving COVID-19 mRNA Vaccine BNT162b2, that were seropositive for SARS-CoV-2 at baseline, was similar to that seen in the general population.

\section{Adolescents 12 to 15 years of age}

In an analysis of Study 2, based on data up to the cut-off date of 13 March 2021, 2,260 adolescents (1,131 COVID-19 mRNA Vaccine BNT162b2; 1,129 placebo) were 12 to 15 years of age. Of these, 1,308 adolescents (660 COVID-19 mRNA Vaccine BNT162b2 and 648 placebo) have been followed for at least 2 months after the second dose of COVID-19 mRNA Vaccine BNT162b2.

The most frequent adverse reactions in adolescents 12 to 15 years of age were injection site pain $(>90 \%)$, fatigue and headache (> 70\%), myalgia and chills (>40\%), arthralgia and pyrexia (> 20\%).

\section{All participants}

The most frequent adverse reactions were usually mild or moderate in intensity and resolved within a few days after vaccination. A slightly lower frequency of reactogenicity events was associated with greater age. If required, symptomatic treatment with analgesic and/or anti-pyretic medicinal products (e.g. paracetamol-containing products) may be used.

Tabulated list of adverse reactions from clinical studies and post-authorisation experience

Adverse reactions observed during clinical studies are listed below according to the following frequency categories:

Very common $(\geq 1 / 10)$,

Common $(\geq 1 / 100$ to $<1 / 10)$,

Uncommon $(\geq 1 / 1,000$ to $<1 / 100)$,

Rare $(\geq 1 / 10,000$ to $<1 / 1,000)$,

Very rare $(<1 / 10,000)$,

Not known (cannot be estimated from the available data). 
Table 1: Adverse reactions from clinical trials and post-authorisation experience

\begin{tabular}{|c|c|c|c|c|c|c|}
\hline $\begin{array}{l}\text { System Organ } \\
\text { Class }\end{array}$ & $\begin{array}{c}\text { Very } \\
\text { common } \\
(\geq 1 / 10)\end{array}$ & $\begin{array}{l}\text { Common } \\
(\geq 1 / 100 \text { to } \\
<1 / 10)\end{array}$ & $\begin{array}{l}\text { Uncommon } \\
(\geq 1 / 1,000 \text { to } \\
<1 / 100)\end{array}$ & $\begin{array}{c}\text { Rare }(\geq \\
1 / 10,000 \\
\text { to }< \\
1 / 1,000)\end{array}$ & $\begin{array}{l}\text { Very rare } \\
(<1 / 10,000)\end{array}$ & $\begin{array}{c}\text { Not known } \\
\text { (cannot be } \\
\text { estimated } \\
\text { from the } \\
\text { available } \\
\text { data) }\end{array}$ \\
\hline $\begin{array}{l}\text { Blood and } \\
\text { lymphatic } \\
\text { system disorders }\end{array}$ & & & Lymphadenopathy & & & \\
\hline $\begin{array}{l}\text { Cardiac } \\
\text { disorders }\end{array}$ & & & & & $\begin{array}{l}\text { Myocarditis } \\
\text { Pericarditis }^{\mathrm{d}}\end{array}$ & \\
\hline $\begin{array}{l}\text { Immune system } \\
\text { disorders }\end{array}$ & & & $\begin{array}{l}\text { Hypersensitivity } \\
\text { reactions (e.g. rash, } \\
\text { pruritus, urticaria, } \\
\text { angioedema }^{\text {b }} \text { ) }\end{array}$ & & & Anaphylaxis \\
\hline $\begin{array}{l}\text { Metabolism and } \\
\text { nutrition } \\
\text { disorders }\end{array}$ & & & Decreased appetite & & & \\
\hline $\begin{array}{l}\text { Psychiatric } \\
\text { disorders }\end{array}$ & & & Insomnia & & & \\
\hline $\begin{array}{l}\text { Nervous system } \\
\text { disorders }\end{array}$ & Headache & & Lethargy & $\begin{array}{l}\text { Acute } \\
\text { peripheral } \\
\text { facial } \\
\text { paralysis }^{c}\end{array}$ & & \\
\hline $\begin{array}{l}\text { Gastrointestinal } \\
\text { disorders }\end{array}$ & Diarrhoea $^{d}$ & $\begin{array}{l}\text { Nausea; } \\
\text { Vomiting }\end{array}$ & & & & \\
\hline $\begin{array}{l}\text { Skin and } \\
\text { subcutaneous } \\
\text { tissue disorder }\end{array}$ & & & $\begin{array}{l}\text { Hyperhidrosis; } \\
\text { Night sweats }\end{array}$ & & & $\begin{array}{l}\text { Erythema } \\
\text { multiforme }\end{array}$ \\
\hline $\begin{array}{l}\text { Musculoskeletal } \\
\text { and connective } \\
\text { tissue disorders }\end{array}$ & $\begin{array}{l}\text { Arthralgia; } \\
\text { Myalgia }\end{array}$ & & Pain in extremity & & & \\
\hline $\begin{array}{l}\text { General } \\
\text { disorders and } \\
\text { administration } \\
\text { site conditions }\end{array}$ & $\begin{array}{l}\text { Injection } \\
\text { site pain; } \\
\text { Fatigue; } \\
\text { Chills; } \\
\text { Pyrexia } \\
\text { Injection } \\
\text { site } \\
\text { swelling }\end{array}$ & $\begin{array}{l}\text { Injection } \\
\text { site } \\
\text { redness }\end{array}$ & $\begin{array}{l}\text { Asthenia; } \\
\text { Malaise; Injection } \\
\text { site pruritus }\end{array}$ & & & $\begin{array}{l}\text { Extensive } \\
\text { swelling of } \\
\text { vaccinated } \\
\text { limb; }{ }^{\text {fFacial }} \\
\text { swelling }{ }^{\mathrm{g}}\end{array}$ \\
\hline
\end{tabular}

a. A higher frequency of lymphadenopathy (5.2\% vs $0.4 \%)$ was observed in participants receiving a booster dose (third dose) compared to participants receiving 2 doses.

b. The frequency category for urticaria and angioedema was Rare.

c. Through the clinical trial safety follow-up period to 14 November 2020, acute peripheral facial paralysis (or palsy) was reported by four participants in the COVID-19 mRNA Vaccine group. Onset was Day 37 after Dose 1 (participant did not receive Dose 2) and Days 3, 9, and 48 after Dose 2. No cases of acute peripheral facial paralysis (or palsy) were reported in the placebo group.

d. Adverse reaction determined post-authorisation.

e. Refers to vaccinated arm.

f. A higher frequency of pyrexia was observed after the second dose compared to the first dose.

g. Facial swelling in vaccine recipients with a history of injection of dermatological fillers has been reported in the post-marketing phase.

Description of selected adverse reactions 


\section{Myocarditis}

The increased risk of myocarditis after vaccination with COVID-19 mRNA Vaccine BNT162b2 is highest in younger males (see section 4.4).

Two large European pharmacoepidemiological studies have estimated the excess risk in younger males following the second dose of COVID-19 mRNA Vaccine BNT162b2. One study showed that in a period of 7 days after the second dose there were about 0.265 (95\% CI $0.255-0.275)$ extra cases of myocarditis in 12-29 year old males per 10,000 compared to unexposed persons. In another study, in a period of 28 days after the second dose there were 0.57 [95\% CI $0.39-0.75$ ] extra cases of myocarditis in 16-24 year old males per 10,000 compared to unexposed persons.

Post-authorisation reports of influenza-like illness

Some recipients have reported chills, shivering (in some cases rigours), and increased body temperature possibly with sweating, headache (including migraine-like headaches), nausea, myalgia and malaise, starting within a day of vaccination. These effects usually last for a day or two.

If a patient reports unusually high and prolonged fever, or other persistent symptoms, alternative causes should be considered and appropriate advice should be provided for diagnostic investigation and medical management as required.

\section{Reporting of suspected adverse reactions}

If you are concerned about an adverse event, it should be reported on a Yellow card. Reporting forms and information can be found at https://coronavirus-yellowcard.mhra.gov.uk/ or search for MHRA Yellow Card in the Google Play or Apple App Store. When reporting please include the vaccine brand and batch/Lot number if available.

Alternatively, adverse events of concern in association with Pfizer BioNTech COVID-19 mRNA vaccine BNT162b2 can be reported to Pfizer Medical Information on 01304616161 or via www.pfizersafetyreporting.com.

Please do not report the same adverse event(s) to both systems as all reports will be shared between Pfizer and MHRA (in an anonymized form) and dual reporting will create unnecessary duplicates.

\subsection{Overdose}

Participants who received 58 micrograms of COVID-19 mRNA Vaccine in clinical trials did not report an increase in reactogenicity or adverse events.

In the event of overdose, monitoring of vital functions and possible symptomatic treatment is recommended.

\section{PHARMACODYNAMIC PROPERTIES}

\subsection{Pharmacodynamic properties}

Pharmacotherapeutic group: vaccines, other viral vaccines, ATC code: J07BX03

Mechanism of action

The nucleoside-modified messenger RNA in COVID-19 mRNA Vaccine BNT162b2 (tozinameran) is formulated in lipid nanoparticles, which enable delivery of the RNA into host cells to allow expression of the SARS-CoV-2 S antigen. The vaccine elicits both neutralising antibody and cellular immune responses to the spike (S) antigen, which may contribute to protection against COVID-19 disease.

\section{Efficacy in participants 16 years of age and older}

The efficacy of COVID-19 mRNA Vaccine BNT162b2 was evaluated in participants 16 years of age and older in two clinical studies conducted in the United States, Europe, Turkey, South Africa and South America. Study 1 enrolled 60 participants, 18 through 55 years of age. Study 2 is a multicentre, 
placebo-controlled efficacy study in participants 12 years of age and older. Randomisation was stratified by age: 12 to 15 years of age, 16 to 55 years of age, or 56 years of age and older, with a minimum of $40 \%$ of participants in the $\geq 56$-year stratum. The study excluded participants who were immunocompromised and those who had previous clinical or microbiological diagnosis of COVID-19 disease. Participants with pre-existing stable disease, defined as disease not requiring significant change in therapy or hospitalisation for worsening disease during the 6 weeks before enrolment, were included as were participants with known stable infection with human immunodeficiency virus (HIV), hepatitis $\mathrm{C}$ virus (HCV) or hepatitis B virus (HBV). There was no requirement for prophylactic use of paracetamol or analgesics. Influenza vaccines could be administered outside a window \pm 14 days of the vaccine doses.

In Study 2, approximately 44,000 participants 12 years of age and older were randomised equally and received 2 doses of COVID-19 mRNA Vaccine or placebo.. The efficacy analyses included participants that received their second vaccination within 19 to 42 days after their first vaccination. Participants are planned to be followed for up to 24 months, for assessments of safety and efficacy against COVID-19 disease.

The population for the analysis of the primary efficacy endpoint included 36,621 participants 12 years of age and older (18,242 in the COVID-19 mRNA Vaccine group and 18,379 in the placebo group) who did not have evidence of prior infection with SARS-CoV-2 through 7 days after the second dose.

Demographic characteristics were generally similar with regard to age, gender, race and ethnicity among participants who received COVID-19 mRNA BNT162b2 vaccine and those who received placebo. Overall, among the participants who received COVID-19 mRNA vaccine, $51.1 \%$ were male and $48.9 \%$ were female, $82.8 \%$ were White, $8.9 \%$ were Black or African American, $26.8 \%$ were Hispanic/Latino, $4.5 \%$ were Asian and $0.6 \%$ were Native American/Alaskan native. $57.2 \%$ were aged $16-55$ years, $42.6 \%$ were aged $>55$ years and $21.8 \%$ were $\geq 65$ years.

The vaccine efficacy information is presented in Table 2 .

Table 2: Vaccine efficacy - First COVID-19 occurrence from 7 days after Dose 2, by age subgroup - participants without evidence of infection prior to 7 days after Dose 2 evaluable efficacy ( 7 days) population

\begin{tabular}{|c|c|c|c|}
\hline \multicolumn{2}{|c|}{ First COVID-19 occurrence from 7 days after Dose 2 in participants without evidence of prior } \\
SARS-CoV-2 infection*
\end{tabular}

Note: Confirmed cases were determined by Reverse Transcription-Polymerase Chain Reaction (RT-PCR) and at least 1 symptom consistent with COVID-19 [*Case definition: (at least 1 of) fever, new or increased cough, new or increased shortness of breath, chills, new or increased muscle pain, new loss of taste or smell, sore throat, diarrhoea or vomiting.]

* Participants who had no serological or virological evidence (prior to 7 days after receipt of the last dose) of past SARS-CoV-2 infection (i.e., N-binding antibody [serum] negative at Visit 1 and SARS-CoV-2 not 
detected by nucleic acid amplification tests (NAAT) [nasal swab] at Visits 1 and 2), and had negative

NAAT (nasal swab) at any unscheduled visit prior to 7 days after Dose 2 were included in the analysis.

a. $\mathrm{N}=$ number of participants in the specified group.

b. $\mathrm{n} 1=$ Number of participants meeting the endpoint definition.

c. Total surveillance time in 1000 person-years for the given endpoint across all participants within each group at risk for the endpoint. Time period for COVID-19 case accrual is from 7 days after Dose 2 to the end of the surveillance period.

d. $\mathrm{n} 2=$ Number of participants at risk for the endpoint.

e. No confirmed cases were identified in adolescents 12 to 15 years of age.

f. Two-sided confidence interval (CI) for vaccine efficacy is derived based on the Clopper and Pearson method adjusted to the surveillance time. CI not adjusted for multiplicity.

Efficacy of COVID-19 mRNA Vaccine in preventing first COVID-19 occurrence from 7 days after Dose 2 compared to placebo was $94.6 \%$ (95\% confidence interval of $89.6 \%$ to $97.6 \%$ ) in participants 16 years of age and older with or without evidence of prior infection with SARS-CoV-2.

There were no meaningful clinical differences in overall vaccine efficacy in participants who were at risk of severe COVID-19 disease including those with one or more comorbidities that increase the risk of severe COVID-19 disease (e.g. asthma, BMI $\geq 30 \mathrm{~kg} / \mathrm{m}^{2}$, chronic pulmonary disease, diabetes mellitus, hypertension).

Updated efficacy analyses were performed with additional confirmed COVID-19 cases accrued during blinded placebo-controlled follow-up, representing up to 6 months after Dose 2 in the efficacy population.

The updated vaccine efficacy information is presented in Table 3.

Table 3: Vaccine efficacy - First COVID-19 occurrence from 7 days after Dose 2, by age subgroup - participants without evidence of prior SARS-CoV-2 infection* prior to 7 days after Dose 2 - evaluable efficacy ( 7 days) population during the placebo-controlled follow-up period

\begin{tabular}{|c|c|c|c|}
\hline Subgroup & $\begin{array}{c}\text { COVID-19 mRNA } \\
\text { Vaccine } \\
\text { Na }^{\mathrm{a}}=\mathbf{2 0 , 9 9 8} \\
\text { Cases } \\
\text { n1 }^{\mathrm{b}} \\
\text { Surveillance time }^{\mathrm{c}}\left(\mathbf{n 2}^{\mathrm{d}}\right)\end{array}$ & $\begin{array}{c}\text { Placebo } \\
\mathbf{N}^{\mathrm{a}}=\mathbf{2 1 , 0 9 6} \\
\text { Cases }^{\mathrm{n}} \\
\text { n1 }^{\mathrm{b}} \\
\text { Surveillance time }^{\mathrm{c}}\left(\mathbf{n 2}^{\mathrm{d}}\right)\end{array}$ & $\begin{array}{c}\text { Vaccine efficacy \% } \\
\left(95 \% \mathbf{C I}^{\mathrm{e}}\right)\end{array}$ \\
\hline All participants ${ }^{\mathrm{f}}$ & $\begin{array}{c}77 \\
6.247(20,712)\end{array}$ & $\begin{array}{c}850 \\
6.003(20,713)\end{array}$ & $\begin{array}{c}91.3 \\
(89.0,93.2)\end{array}$ \\
\hline 16 to 64 years & $\begin{array}{c}70 \\
4.859(15,519)\end{array}$ & $\begin{array}{c}710 \\
4.654(15,515)\end{array}$ & $\begin{array}{c}90.6 \\
(87.9,92.7)\end{array}$ \\
\hline 65 years and older & $\begin{array}{c}7 \\
1.233(4192)\end{array}$ & $\begin{array}{c}124 \\
1.202(4226)\end{array}$ & $\begin{array}{c}94.5 \\
(88.3,97.8)\end{array}$ \\
\hline 65 to 74 years & $\begin{array}{c}6 \\
0.994(3350)\end{array}$ & $\begin{array}{c}98 \\
0.966(3379)\end{array}$ & $\begin{array}{c}94.1 \\
(86.6,97.9)\end{array}$ \\
\hline 75 years and older & $\begin{array}{c}1 \\
0.239(842)\end{array}$ & $\begin{array}{c}26 \\
0.237(847)\end{array}$ & $\begin{array}{c}96.2 \\
(76.9,99.9)\end{array}$ \\
\hline
\end{tabular}

Note: Confirmed cases were determined by Reverse Transcription-Polymerase Chain Reaction (RT-PCR) and at least 1 symptom consistent with COVID-19 (symptoms included: fever; new or increased cough; new or increased shortness of breath; chills; new or increased muscle pain; new loss of taste or smell; sore throat; diarrhoea; vomiting).

* Participants who had no evidence of past SARS-CoV-2 infection (i.e., N-binding antibody [serum] negative at Visit 1 and SARS-CoV-2 not detected by NAAT [nasal swab] at Visits 1 and 2), and had negative NAAT (nasal swab) at any unscheduled visit prior to 7 days after Dose 2 were included in the analysis.

a. $\mathrm{N}=$ Number of participants in the specified group.

b. $\mathrm{n} 1=$ Number of participants meeting the endpoint definition. 
Table 3: Vaccine efficacy - First COVID-19 occurrence from 7 days after Dose 2, by age subgroup - participants without evidence of prior SARS-CoV-2 infection* prior to 7 days after Dose 2 - evaluable efficacy ( 7 days) population during the placebo-controlled follow-up period

c. Total surveillance time in 1,000 person-years for the given endpoint across all participants within each group at risk for the endpoint. Time period for COVID-19 case accrual is from 7 days after Dose 2 to the end of the surveillance period.

d. $\quad \mathrm{n} 2=$ Number of participants at risk for the endpoint.

e. Two-sided 95\% confidence interval (CI) for vaccine efficacy is derived based on the Clopper and Pearson method adjusted to the surveillance time.

f. Included confirmed cases in participants 12 to 15 years of age: 0 in the COVID-19 mRNA Vaccine group; 16 in the placebo group.

In the updated efficacy analysis, efficacy of COVID-19 mRNA Vaccine in preventing first COVID-19 occurrence from 7 days after Dose 2 compared to placebo was $91.1 \%$ (95\% CI of $88.8 \%$ to $93.0 \%$ ) in participants in the evaluable efficacy population with or without evidence of prior infection with SARS-CoV-2.

Additionally, the updated efficacy analyses by subgroup showed similar efficacy point estimates across sexes, ethnic groups, geography and participants with medical comorbidities and obesity associated with high risk of severe COVID-19.

Efficacy against severe COVID-19

Updated efficacy analyses of secondary efficacy endpoints supported benefit of the COVID-19 mRNA Vaccine in preventing severe COVID-19.

As of 13 March 2021, vaccine efficacy against severe COVID-19 is presented only for participants with or without prior SARS-CoV-2 infection (Table 3) as the COVID-19 case counts in participants without prior SARS-CoV-2 infection were the same as those in participants with or without prior SARS-CoV-2 infection in both the COVID-19 mRNA Vaccine and placebo groups.

Table 4: Vaccine efficacy - First severe COVID-19 occurrence in participants with or without prior SARS-CoV-2 infection based on the Food and Drug Administration (FDA)* after Dose 1 or from 7 days after Dose 2 in the placebo-controlled follow-up

\begin{tabular}{|c|c|c|c|}
\hline & $\begin{array}{c}\text { COVID-19 mRNA } \\
\text { Vaccine } \\
\text { Cases } \\
\text { n1 }^{\mathrm{a}} \\
\text { Surveillance time }\left(\mathbf{n} 2^{\mathrm{b}}\right)\end{array}$ & $\begin{array}{c}\text { Placebo } \\
\text { Cases } \\
\text { n1 }{ }^{\mathbf{a}} \\
\text { Surveillance time }\left(\mathbf{n} 2^{\mathbf{b}}\right)\end{array}$ & $\begin{array}{c}\text { Vaccine efficacy \% } \\
\left(95 \% \mathbf{C I}^{\mathbf{c}}\right)\end{array}$ \\
\hline After Dose $1^{\mathrm{d}}$ & $\begin{array}{c}1 \\
8.439^{\mathrm{e}}(22,505)\end{array}$ & $\begin{array}{c}30 \\
8.288^{\mathrm{e}}(22,435)\end{array}$ & $\begin{array}{c}96.7 \\
(80.3,99.9)\end{array}$ \\
\hline 7 days after Dose $2^{f}$ & $\begin{array}{c}1 \\
6.522^{\mathrm{g}}(21,649)\end{array}$ & $\begin{array}{c}21 \\
6.404^{\mathrm{g}}(21,730)\end{array}$ & $\begin{array}{c}95.3 \\
(70.9,99.9)\end{array}$ \\
\hline
\end{tabular}

Note: Confirmed cases were determined by Reverse Transcription-Polymerase Chain Reaction (RT-PCR) and at least 1 symptom consistent with COVID-19 (symptoms included: fever; new or increased cough; new or increased shortness of breath; chills; new or increased muscle pain; new loss of taste or smell; sore throat; diarrhoea; vomiting).

* Severe illness from COVID-19 as defined by FDA is confirmed COVID-19 and presence of at least 1 of the following:

- Clinical signs at rest indicative of severe systemic illness (respiratory rate $\geq 30$ breaths per minute, heart rate $\geq 125$ beats per minute, saturation of oxygen $\leq 93 \%$ on room air at sea level, or ratio of arterial oxygen partial pressure to fractional inspired oxygen $<300 \mathrm{~mm} \mathrm{Hg}$ );

- Respiratory failure [defined as needing high-flow oxygen, noninvasive ventilation, mechanical ventilation or extracorporeal membrane oxygenation (ECMO)];

- Evidence of shock (systolic blood pressure $<90 \mathrm{~mm} \mathrm{Hg}$, diastolic blood pressure $<60 \mathrm{~mm} \mathrm{Hg}$, or requiring vasopressors);

- Significant acute renal, hepatic, or neurologic dysfunction; 
Table 4: Vaccine efficacy - First severe COVID-19 occurrence in participants with or without prior SARS-CoV-2 infection based on the Food and Drug Administration (FDA)* after Dose 1 or from 7 days after Dose 2 in the placebo-controlled follow-up

- Admission to an Intensive Care Unit;

- Death.

a. $n 1=$ Number of participants meeting the endpoint definition.

b. $\mathrm{n} 2=$ Number of participants at risk for the endpoint.

c. Two-side confidence interval (CI) for vaccine efficacy is derived based on the Clopper and Pearson method adjusted to the surveillance time.

d. Efficacy assessed based on the Dose 1 all available efficacy (modified intention-to-treat) population that included all randomised participants who received at least 1 dose of study intervention.

e. Total surveillance time in 1,000 person-years for the given endpoint across all participants within each group at risk for the endpoint. Time period for COVID-19 case accrual is from Dose 1 to the end of the surveillance period.

f. Efficacy assessed based on the evaluable efficacy (7 Days) population that included all eligible randomised participants who receive all dose(s) of study intervention as randomised within the predefined window, have no other important protocol deviations as determined by the clinician.

g. Total surveillance time in 1,000 person-years for the given endpoint across all participants within each group at risk for the endpoint. Time period for COVID-19 case accrual is from 7 days after Dose 2 to the end of the surveillance period.

\section{Efficacy and immunogenicity in adolescents 12 to 15 years of age}

An analysis of Study 2 has been performed in adolescents 12 to 15 years of age up to a data cut-off date of 13 March 2021.

The vaccine efficacy information in adolescents 12 to 15 years of age is presented in Table 5 .

Table 5: Vaccine efficacy - First COVID-19 occurrence from 7 days after Dose 2 participants without evidence of infection and with or without evidence of infection prior to 7 days after Dose 2 - adolescents 12 to 15 years of age evaluable efficacy (7 days) population

\begin{tabular}{|l|c|c|c|}
\hline \multicolumn{4}{|c|}{ First COVID-19 occurrence from 7 days after Dose 2 in adolescents 12 to 15 years of age } \\
without evidence of prior SARS-CoV-2 infection*
\end{tabular}

Note: Confirmed cases were determined by Reverse Transcription-Polymerase Chain Reaction (RT-PCR) and at least 1 symptom consistent with COVID-19 [*Case definition: (at least 1 of) fever, new or increased cough, new or increased shortness of breath, chills, new or increased muscle pain, new loss of taste or smell, sore throat, diarrhoea or vomiting).

* Participants who had no serological or virological evidence (prior to 7 days after receipt of the last dose) of past SARS-CoV-2 infection (i.e, N-binding antibody [serum] negative at Visit 1 and SARS-CoV-2 not 
detected by nucleic acid amplification tests (NAAT) [nasal swab] at Visits 1 and 2), and had negative NAAT (nasal swab) at any unscheduled visit prior to 7 days after Dose 2 were included in the analysis.

a. $\mathrm{N}=$ number of participants in the specified group.

b. $\mathrm{n} 1=$ Number of participants meeting the endpoint definition.

c. Total surveillance time in 1000 person-years for the given endpoint across all participants within each group at risk for the endpoint. Time period for COVID-19 case accrual is from 7 days after Dose 2 to the end of the surveillance period.

d. $\mathrm{n} 2=$ Number of participants at risk for the endpoint.

e. Confidence interval (CI) for vaccine efficacy is derived based on the Clopper and Pearson method adjusted for surveillance time. CI not adjusted for multiplicity.

In Study 2 an analysis of SARS-CoV-2 neutralising titres in a randomly selected subset of participants was performed to demonstrate non-inferior immune responses (within 1.5-fold) comparing adolescents 12 to 15 years of age to participants 16 to 25 years of age who had no serological or virological evidence of past SARS-CoV-2 infection. The immune response to COVID-19 mRNA Vaccine BNT162b2 in adolescents 12 to 15 years of age $(n=190)$ was non-inferior to the immune response in participants 16 to 25 years of age $(n=170)$, based on results for SARS-CoV-2 neutralising titres at 1 month after Dose 2. The geometric mean titres (GMT) ratio of the adolescents 12 to 15 years of age group to the participants 16 to 25 years of age group was 1.76 , with a 2-sided $95 \%$ CI of 1.47 to 2.10 , meeting the 1.5-fold non-inferiority criterion (the lower bound of the 2-sided 95\% CI for the geometric mean ratio $[\mathrm{GMR}]>0.67$ ) and indicating a statistically greater response in the adolescents 12 to 15 years of age than that of participants 16 to 25 years of age.

\section{Immunogenicity in immunocompromised patients}

From an independent report (Kamar N, Abravanel F, Marion O, et al. Three doses of an mRNA Covid19 vaccine in solid-organ transplant recipients. $N$ Engl J Med. 2021), a single arm study has been conducted in 101 individuals who had undergone various solid organ transplant procedures (heart, kidney, liver, lung, pancreas) $97 \pm 8$ months previously. A third dose of COVID-19 mRNA Vaccine BNT162b2 was administered to 99 of these individuals approximately 2 months after they had received a second dose. Among the 59 patients who had been seronegative before the third dose, 26 (44\%) were seropositive at 4 weeks after the third dose. All 40 patients who had been seropositive before the third dose were still seropositive 4 weeks later. The prevalence of anti-SARS-CoV-2 antibodies was $68 \%$ (67 of 99 patients) 4 weeks after the third dose.

There are no data in immunocompromised patients other than those with solid organ transplant.

\section{Immunogenicity of a booster dose in adults}

In Phase 1 of Study 2,11 subjects aged 24 to 55 years and 12 subjects aged 65 to 75 years received a third dose of vaccine after a median of 8 months following the second dose. In both age groups, neutralising antibody GMTs measured 28 days after the third dose against the Wuhan strain as well as beta and delta variants were all > 5-fold those of GMTs measured 28 days after the second dose.

In Phase 3 of Study 2, a cohort of 306 participants, 18 - 55 years old (median 42 years), received a third dose after a median of 7 months (range: 4.8 - 8.0) following the second dose; $40 \%$ were obese. Effectiveness of the third dose was demonstrated by evaluating noninferiority of the immune response of neutralising titres (NT50) against the Wuhan strain 1 month after the third dose compared to 1 month after the second dose in individuals who had no serological or virological evidence of past SARS CoV 2 infection up to 1 month after the third dose. The day 28 post-dose GMT ratio of the third to the second dose was 3.29 (2-sided 97.5\%CI: 2.76, 3.91), showing a statistically greater response following the third dose compared to the second dose. The seroresponse rate, defined as the proportion of subjects achieving a $\geq 4$-fold rise from baseline (before the first dose), was $99.5 \%$ after the third dose vs $98.0 \%$ after the second dose, i.e. a difference of $1.5 \%$ (2-sided $97.5 \% \mathrm{CI}$ : $-0.7,3.7 \%$ ); this met the $10 \%$ noninferiority criterion (i.e., lower bound of the 2 -sided $97.5 \%$ CI > - 10\%).

In these two sub-studies, the reactogenicity profile after the third dose was comparable to that after the second dose. The most common local reaction was injection site pain and the most common systemic 
reactions included fatigue, headache, muscle and joint pain, and chills. The frequency of severe systemic reactions was low, less than $2 \%$ of participants for all systemic reactions except for severe fatigue (4.5\%). Systemic reactions resolved within a median duration of 1 to 2 days.

The most commonly reported adverse event was lymphadenopathy, in 16/306 participants (5.2\%), which was in all cases considered by the investigator as related to the vaccine.

Additional data assessed by the MHRA that support the use of COVID-19 mRNA Vaccine BNT162b2 as a heterologous booster dose are based on the COV-Boost study [EudraCT Number: 2021-00217519), which investigated homologous and different combination of heterologous boosters following primary immunisation against COVID-19. In total, 110 participants were given COVID-19 mRNA Vaccine BNT162b2 as a homologous booster at a median of 100 days (3.3 months) after the second dose and 107 participants as a heterologous boosters at a median of 77 days ( 2.5 months) following the second dose of an adenovirus-vectored vaccine. A several-fold increase in binding and neutralising antibodies as well as T-cell response pre/post third dose was observed in both arms. The MHRA has needed to rely on these data in advance of them being publicly available, including to Pfizer/BioNTech, but is satisfied as to the arrangements for its expected publication, and this section will be updated once the data are published.

There are no data on a booster dose administered to individuals less than 18 years old.

\subsection{Pharmacokinetic properties}

Not applicable.

\subsection{Preclinical safety data}

Non-clinical data reveal no special hazard for humans based on a conventional study of repeat dose toxicity.

$\underline{\text { Reproductive toxicity }}$

Reproductive and developmental toxicity were investigated in rats in a combined fertility and developmental toxicity study where female rats were intramuscularly administered with the COVID19 mRNA Vaccine BNT162b2 prior to mating and during gestation (receiving 4 full human doses that generate relatively higher levels in rat due to body weight differences, spanning between pre-mating day 21 and gestational day 20). SARS-CoV-2 neutralizing antibody responses were present in maternal animals from prior to mating to the end of the study on postnatal day 21 as well as in foetuses and offspring. There were no vaccine-related effects on female fertility, pregnancy, or embryo-foetal or offspring development. No data on the COVID-19 mRNA Vaccine BNT162b2 are available on vaccine placental transfer or excretion in milk.

\section{PHARMACEUTICAL PARTICULARS}

\subsection{List of excipients}

This vaccine contains polyethylene glycol/macrogol (PEG) as part of ALC-0159.

ALC-0315 = (4-hydroxybutyl) azanediyl)bis (hexane-6,1-diyl)bis(2-hexyldecanoate), ALC-0159 = 2-[(polyethylene glycol)-2000]-N,N-ditetradecylacetamide,

1,2-Distearoyl-sn-glycero-3-phosphocholine,

cholesterol,

potassium chloride,

potassium dihydrogen phosphate,

sodium chloride,

disodium hydrogen phosphate dihydrate, 
sucrose,

water for injections

\subsection{Incompatibilities}

In the absence of compatibility studies, this medicinal product must not be mixed with other medicinal products.

\subsection{Shelf life}

9 months at $-80^{\circ} \mathrm{C}$ to $-60^{\circ} \mathrm{C}$.

\subsection{Special precautions for storage}

Store in a freezer at $-80^{\circ} \mathrm{C}$ to $-60^{\circ} \mathrm{C}$.

Store in the thermal container at $-90^{\circ} \mathrm{C}$ to $-60^{\circ} \mathrm{C}$.

Store in the original package in order to protect from light.

Once removed from the freezer, the undiluted vaccine can be stored for up to 1 month ( 31 days) at $2^{\circ} \mathrm{C}$ to $8^{\circ} \mathrm{C}$, and up to 2 hours at temperatures up to $25^{\circ} \mathrm{C}$, prior to use. During storage, minimise exposure to room light, and avoid exposure to direct sunlight and ultraviolet light. Thawed vials can be handled in room light conditions.

After dilution, store the vaccine at $2^{\circ} \mathrm{C}$ to $25^{\circ} \mathrm{C}$ and use as soon as practically possible and within 6 hours. The vaccine does not contain a preservative. Discard any unused vaccine.

Once diluted, the vials should be marked with the dilution time and discarded within 6 hours of dilution.

Once thawed, the vaccine cannot be re-frozen.

\subsection{Nature and contents of container}

Concentrate for solution for injection for 6 doses in a $2 \mathrm{~mL}$ clear vial (type I glass) with a stopper (bromobutyl) and a flip-off plastic cap with aluminium seal, see section 4.2.

Pack size: 195 vials

\subsection{Special precautions for disposal and other handling}

When removed from the freezer, the vaccine has a maximum possible shelf life of up to 1 month (31 days) when stored at $2-8^{\circ} \mathrm{C}$ (label to be added once box removed from freezer). A 195 vial pack may take 3 hours to thaw at $2-8^{\circ} \mathrm{C}$.

The product can alternatively be defrosted and kept for up to 2 hours at up to $25^{\circ} \mathrm{C}$ before being diluted for use. This facilitates immediate thaw and use when removed directly from the freezer to $25^{\circ} \mathrm{C}$. In this instance the product is to be diluted within 2 hours of removing from the freezer.

Once thawed, the vaccine cannot be refrozen.

After dilution the vaccine should be used as soon as is practically possible and within 6 hours of dilution; it can be stored at $2-25^{\circ} \mathrm{C}$ during this period. From a microbiological point of view, it would not normally be considered good practice to store a diluted product for 6 hours at $25^{\circ} \mathrm{C}$ before being administered. The product would ideally be used as soon as practically possible after dilution.

The vaccine does not contain a preservative. Discard any unused vaccine. 
Any unused medicinal product or waste material should be disposed of in accordance with local requirements.

For instructions on dose preparation of the medicinal product before administration, see section 4.2.

\section{MARKETING AUTHORISATION HOLDER}

Not applicable.

\section{MARKETING AUTHORISATION NUMBER(S)}

Not applicable.

\section{DATE OF FIRST AUTHORISATION/RENEWAL OF THE AUTHORISATION}

Not applicable.

\section{DATE OF REVISION OF THE TEXT}

December 2021 